\title{
Effects of ghrelin on the apoptosis of human neutrophils in vitro
}

\author{
BIN LI ${ }^{*}$, MIAN ZENG ${ }^{*}$, HAICHONG ZHENG, CHUNRONG HUANG, WANMEI HE, \\ GUIFANG LU, XIA LI, YANZHU CHEN and RUIJIE XIE

\begin{abstract}
Department of Medical Intensive Care Unit, The First Affiliated Hospital, Sun Yat-Sen University, Guangzhou, Guangdong 510080, P.R. China
\end{abstract}

Received October 22, 2015; Accepted June 30, 2016

DOI: $10.3892 / \mathrm{ijmm} .2016 .2668$

\begin{abstract}
Acute respiratory distress syndrome (ARDS) is characterized by lung inflammation and the diffuse infiltration of neutrophils into the alveolar space. Neutrophils are abundant, short-lived leukocytes that play a key role in immune defense against microbial infections. These cells die via apoptosis following the activation and uptake of microbes, and will also enter apoptosis spontaneously at the end of their lifespan if they do not encounter pathogens. Apoptosis is essential for the removal of neutrophils from inflamed tissues and for the timely resolution of neutrophilic inflammation. Ghrelin is an endogenous ligand for the growth hormone (GH) secretagogue receptor, produced and secreted mainly from the stomach. Previous studies have reported that ghrelin exerts anti-inflammatory effects in lung injury through the regulation of the apoptosis of different cell types; however, the ability of ghrelin to regulate alveolar neutrophil apoptosis remains largely undefined. We hypothesized that ghrelin may have the ability to modulate neutrophil apoptosis. In this study, to examine this hypothesis, we investigated the effects of ghrelin on freshly isolated neutrophils in vitro. Our findings demonstrated a decrease in the apoptotic ratio (as shown by flow cytometry), as well as in the percentage of cells with decreased mitochondrial membrane potential $(\Delta \Psi \mathrm{m})$ and in the terminal deoxynucleotidyl transferase (TdT)-mediated dUTP-biotin nick-end labeling-positive rate, accompanied by an increased B-cell lymphoma 2/Bax ratio and the downregulation of cleaved caspase- 3 in neutrophils following exposure to lipopolysaccharide $(100 \mathrm{ng} / \mathrm{ml})$. However, pre-treatment with ghrelin at a physiological level $(100 \mathrm{nM})$ did not have a notable influence on the neutrophils in all the aforementioned tests.
\end{abstract}

Correspondence to: Professor Mian Zeng, Department of Medical Intensive Care Unit, The First Affiliated Hospital, Sun Yat-Sen University, 58 Zhongshan 2nd Road, Guangzhou, Guangdong 510080, P.R. China

E-mail: zengmian2004@163.com

*Contributed equally

Key words: ghrelin, lipopolysaccharide, apoptosis, neutrophils, acute respiratory distress syndrome
Our findings suggest that ghrelin may not possess the ability to modulate the neutrophil lifespan in vitro.

\section{Introduction}

Acute respiratory distress syndrome (ARDS) is characterized by overwhelming lung inflammation and increased microvascular permeability, causing the diffuse infiltration of neutrophils and lung edema (1). Neutrophils, rapidly recruited from the circulation to sites of infection or tissue injury, play a prominent role in host defense against invading pathogens (2). The effective resolution of inflammation and the restoration of tissue homeostasis critically depends on the neutrophil influx and the timely removal of infiltrating neutrophils (3). However, as these neutrophil functions lack specificity and can be injurious to host tissues, it is important that neutrophil activity be tightly regulated to prevent the perpetuation of inflammation. Actually, neutrophil apoptosis can be delayed at infection sites by a range of factors, including bacterial products such as lipopolysaccharide (LPS) (4), and pro-inflammatory cytokines (5), as well as hypoxia (6), ostensibly to extend their functional lifespan (7). Once an episode of acute neutrophilic inflammation is complete, it is essential that neutrophil recruitment is halted and that recruited neutrophils undergo apoptosis, before disposal of the apoptotic cells by surrounding phagocytes, such as alveolar macrophages $(3,8)$, to ensure the efficient resolution of inflammation (9). However, in reality, the natural resolution process of neutrophil apoptosis followed by phagocytosis is impaired in numerous human inflammatory disease states, with delayed neutrophil apoptosis observed in conditions such as ARDS (10). Observations in medical patients showed that neutrophil apoptosis is inversely proportional to the severity of sepsis and sepsis-induced ARDS, suggesting that neutrophil apoptosis may be a marker of the severity of sepsis (10). Furthermore, accumulating evidence has indicated a consistent association between neutrophils and ARDS in human and animal models and the propensity of neutrophils and their products to cause lung injury in experimental systems, leading to the conclusion that neutrophils have an important causative role in ARDS, and neutrophil apoptosis could emerge as a critical control point in resolving inflammation. Optimistically, recent studies using a variety of gene knockout, transgenic and pharmacological strategies in diverse models of inflammation, have demonstrated that modulating neutrophil apoptosis can profoundly affect the outcome of inflammation (11-15). 
Ghrelin is a 28-amino-acid peptide esterified with octanoic acid on Ser 3 that is principally released from the stomach mucosa (16). Apart from its regulatory roles in growth hormone release, appetite and gastric motility, ghrelin also has numerous peripheral actions, including anti-inflammatory, anti-fibrotic and antioxidant properties $(16,17)$. The administration of ghrelin has been reported to attenuate neutrophil migration, reduce inflammatory cytokine production, promote the generation of anti-inflammatory cytokines, and promote the phagocytosis of apoptotic neutrophils by macrophages $(16,17)$, thereby attenuating inflammation in several disease models, including respiratory infection (18), polymicrobial sepsis $(19,20)$, arthritis (21) and ARDS (20,22). However, little is known about whether ghrelin is able to regulate neutrophil apoptosis, a critical control point in the resolution of inflammation.

Based on previous observations, we hypothesized that ghrelin may ameliorate lung impairment in ARDS by promoting neutrophil apoptosis. Therefore, in this study, we examined this hypothesis by investigating the role of ghrelin in ARDS using primary cultured human peripheral blood neutrophils. We examined the effects of ghrelin on neutrophil apoptosis using flow cytometry, as well as by performing mitochondrial membrane potential $(\Delta \Psi \mathrm{m})$ and terminal deoxynucleotidyl transferase (TdT)-mediated dUTP nick-end labeling (TUNEL) assays. We also performed western blot analysis and immunochemistry to detect the expression of B-cell lymphoma 2 (Bcl-2) family proteins and cleaved caspase-3. Our study aimed at providing laboratory evidence and a potential theoretical basis for the effects of ghrelin on neutrophil apoptosis in order to further elucidate the molecular mechanisms responsible for the anti-inflammatory function of ghrelin in ARDS.

\section{Materials and methods}

Reagents and antibodies. Human recombinant ghrelin was purchased from Enzo Life Sciences (Farmingdale, NY, USA). Percoll was purchased from Axis-Shield (Oslo, Norway). RPMI-1640 medium, fetal bovine serum (FBS), and penicillin-streptomycin were obtained from Life Technologies (Grand Island, NY, USA). Dimethyl sulfoxide (DMSO), 4',6-diamidino-2-phenylindole (DAPI), Hoechst 33342, Tween-20, and all buffers and salt solutions were purchased from Sigma-Aldrich (St. Louis, MO, USA). The TUNEL assay kit, protease inhibitor cocktail, and phosphatase inhibitor cocktails were from Roche (Basel, Switzerland). The cleaved caspase-3 antibody (\#AB3623) was from Millipore (Billerica, MA, USA), and the Annexin V-fluorescein isothiocyanate (FITC) cell apoptosis kit was obtained from BD Biosciences (San Jose, CA, USA). Antibodies against Bax (\#2772), Bcl-2 (\#2876), GAPDH (\#2118) and peroxidase-conjugated secondary antibody (\#7074) were all purchased from Cell Signaling Technology, Inc. (Danvers, MA, USA). Alexa Fluor-labeled secondary antibody (\#R37167) was purchased from Invitrogen (Carlsbad, CA, USA).

Neutrophil isolation and treatment. Venous blood collected from 20 healthy subjects was fractionated on Ficoll-Hypaque and dextran gradients to separate neutrophils. All blood samples were collected after obtaining written informed consent from each subject and following the approval of the Medical Ethics Committe of the First Affiliated Hospital, Sun Yat-Sen
University, Guangzhou, China. The neutrophil layer was then transferred to a fresh tube and centrifuged for $10 \mathrm{~min}$ at $4^{\circ} \mathrm{C}$. Hypotonic lysis with $0.2 \% \mathrm{NaCl}$ was performed to remove the remaining erythrocytes. The morphology and purity of the neutrophils were assessed by Giemsa (Beijing Leagene Biotech Co., Ltd., Beijing, China) and Hoechst staining. For all experiments, isolated neutrophils were resuspended in RPMI1640 medium, containing $100 \mathrm{U} / \mathrm{ml}$ penicillin and $100 \mu \mathrm{g} /$ $\mathrm{ml}$ streptomycin supplemented with $10 \%$ FBS. The prepared neutrophils were divided into the following groups: i) the control group (neutrophils + medium); ii) the LPS group (neutrophils + medium + $100 \mathrm{ng} / \mathrm{ml}$ LPS); iii) the LPS + ghrelin group (neutrophils + medium + $100 \mathrm{ng} / \mathrm{ml}$ LPS + $100 \mathrm{nM}$ ghrelin); and iv) the ghrelin group (neutrophils + medium $+100 \mathrm{nM}$ ghrelin). The isolated human neutrophils were incubated with or without ghrelin $(100 \mathrm{nM})$ for $1.5 \mathrm{~h}$ prior to the addition of LPS $(100 \mathrm{ng} / \mathrm{ml})$ for $8 \mathrm{~h}$. The concentrations of the drugs were selected based on previous studies $(23,37)$ and on preliminary experiments (data not shown).

Assessment of neutrophil apoptosis. Apoptosis was determined by 3 methods. Apoptosis was first evaluated by cytofluorometry using the Annexin V-FITC kit. Briefly, the cells in the different treatment groups were centrifuged at $100 \mathrm{x} g$ for $5 \mathrm{~min}$, washed twice with cold phosphate-buffered saline (PBS), and then resuspended in $500 \mu \mathrm{l}$ binding buffer. The cells were then labeled with Annexin V-FITC (5 $\mu \mathrm{l})$ and propidium iodide (PI, $5 \mu \mathrm{l}$ ) for $15 \mathrm{~min}$ in the dark at room temperature before being analyzed using a FACScan flow cytometer (BD Biosciences).

The collapse of the electrochemical gradient across the mitochondrial membrane during apoptosis was measured using the JC-1 mitochondrial membrane potential detection kit (Beyotime, Beijing, China) using flow cytometry as described by the manufacturer. This kit uses a unique cationic dye, JC-1, to signal the loss of the $\Delta \Psi \mathrm{m}$. Briefly, following treatment with the indicated agents and concentrations for $8 \mathrm{~h}$, the cells were collected, resuspended in JC-1 reagent solution, and incubated for $15 \mathrm{~min}$ at $37^{\circ} \mathrm{C}$ in the dark. The cells were then washed with a buffer solution and resuspended in assay buffer, and analyzed by a FACScan flow cytometer.

To measure the double-stranded cleavage of DNA, TUNEL assay was performed with an in situ cell death detection kit following the manufacturer's instructions. Briefly, the cells grown on coverslips were washed with PBS and fixed with $4 \%$ paraformaldehyde for $15 \mathrm{~min}$ at room temperature. After washing, the cells were incubated in permeabilization solution $(0.1 \%$ Triton $\mathrm{X}-100$ in $0.1 \%$ sodium citrate, freshly prepared) for $2 \mathrm{~min}$ on ice. The cells were then incubated with the TUNEL reaction mixture in a humidified chamber at $37^{\circ} \mathrm{C}$ for $1 \mathrm{~h}$. Subsequently, the cells were briefly rinsed with PBS and counterstained with DAPI for $5 \mathrm{~min}$ in order to visualize the nuclei. Stained sections were examined by a fluorescence microscope (Carl Zeiss, Tokyo, Japan), and images of 5 random and non-overlapping fields were examined at an objective magnification of x200 for analysis.

Western blot analysis. Following BCA protein assaying (Thermo Fisher Scientific, Cramlington, UK), equal amounts of proteins were separated by $10 \%$ sodium dodecyl sulfate-polyacrymide gel electrophoresis and transferred onto nitrocellulose 

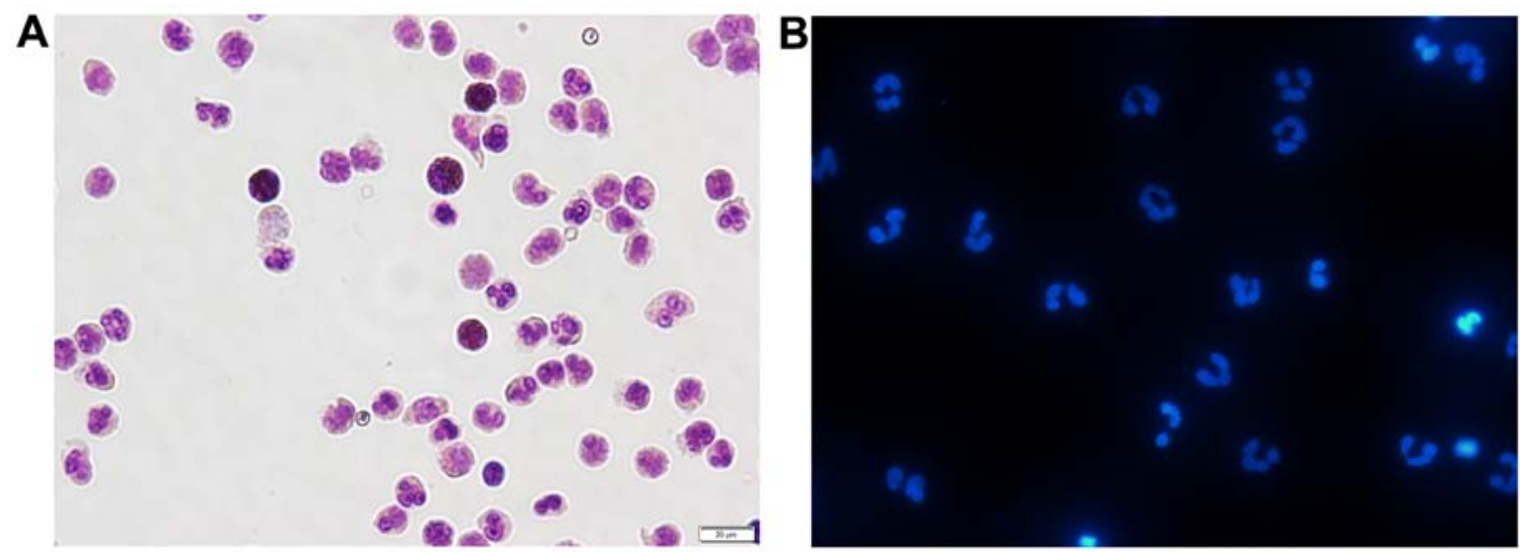

Figure 1. Highly purified population of freshly isolated peripheral blood neutrophils (x400 magnification). (A) Peripheral blood neutrophils from healthy subjects displayed normal mature morphology after Giemsa staining. Neutrophils determined to be approximately $95 \%$ of all cells. (B) Peripheral blood neutrophils were stained with Hoechst 33342 dye to visualize the typical nuclear morphology of neutrophils.

membranes, which were blocked using $5 \%$ non-fat milk for $1 \mathrm{~h}$ at room temperature. The membranes were then incubated overnight at $4^{\circ} \mathrm{C}$ with primary antibodies specific for Bax $(1: 1,000$ dilution), Bcl-2 (1:1,000 dilution), or cleaved caspase-3 (1:200 dilution). After washing, the membranes were incubated with horseradish peroxidase-conjugated anti-mouse or anti-rabbit secondary antibodies for $1 \mathrm{~h}$ at room temperature. Proteins were visualized using an enhanced chemiluminescence kit (Millipore). The relative protein expression levels were determined by densitometry and normalized to the GAPDH levels using Image Pro Plus 6.0 software (Media Cybernetics, Rockville, MD, USA).

Detection of cleaved caspase-3 by immunofluorescence. The cells grown on coverslips were fixed with paraformaldehyde for $15 \mathrm{~min}$ at room temperature. After washing with PBS 3 times for $5 \mathrm{~min}$, the cells were incubated for $30 \mathrm{~min}$ with $1 \%$ bovine serum albumin (BSA) to block the non-specific binding of the antibodies. The cells were then incubated with primary antibody (1:200 dilution) in 1\% BSA in a humidified chamber overnight at $4^{\circ} \mathrm{C}$. After washing 3 times in PBS, the cell samples were incubated with Alexa Fluor 488-conjugated goat antirabbit IgG secondary antibody (1:200) in $1 \%$ BSA for $1 \mathrm{~h}$ in the dark at room temperature, and then washed and counterstained with DAPI for $5 \mathrm{~min}$. The coverslips were then visualized under a fluorescence microscope at x200 magnification. Quantification was performed using Image Pro Plus 6.0 software.

Statistical analyses. Statistical significance of the differences between groups among repeated experiments was calculated by one-way ANOVA and Fisher's LSD-test using GraphPad Prism 4 software (GraphPad Software Ltd, La Jolla, CA, USA). The results are expressed as the mean values \pm standard devitation. In all statistical tests, a P-value $<0.05$ was considered to indicate a statistically significant difference.

\section{Results}

Determination of the viability and purity of the isolated neutrophils by Giemsa and Hoechst staining. The use of a percoll gradient to purify peripheral blood neutrophils has been previ- ously described (24). This approach results in a highly purified population of neutrophils, with a purity $>95 \%$ (as shown by Giemsa staining) and a viability $>98 \%$ (as shown by trypan blue staining) (data not shown). As shown in Fig. 1A, after Giemsa staining, normal cell nuclei were dyed blue or hyacinthine, with homogeneous color and luster. Hoechst 33342 staining revealed a typical nuclear morphology in the freshly isolated neutrophils (Fig. 1B). The examination of cell purity proved that the isolated neutrophils perfectly met the requirements for all the subsequent experiments.

Detection of neutrophil apoptosis by flow cytometry. The occurrence of neutrophil apoptosis was demonstrated using the Annexin V-FITC staining methods (Fig. 2). The results of flow cytometry assay revealed that the ratio of apoptotic cells significantly decreased from $38.67 \pm 2.09$ in the untreated cells to $20.80 \pm 1.09 \%$ and $18.57 \pm 2.32 \%$ in the LPS-stimulated cells and LPS + ghrelin-treated cells, respectively, indicating that LPS significantly prolonged the neutrophil lifespan. However, treatment with ghrelin neither induced nor inhibited neutrophil apoptosis, since there were no apparent differences observed in the percentages of apoptotic neutrophils between the LPS group and the LPS + ghrelin group, and between the control and ghrelin groups (32.39 $\pm 1.84 \%)$ (Fig. 2).

Detection of neutrophil apoptosis by $\Delta \Psi m$ assay. The depolarization of $\Delta \Psi \mathrm{m}$ is implicated in apoptosis (25). Therefore, we then measured $\Delta \Psi \mathrm{m}$ using JC-1 staining. As shown in Fig. 3, the percentage of cells that lost their $\Delta \Psi \mathrm{m}$ in the LPS group was markedly decreased compared to the control group, indicating that LPS was helpful in sustaining a stable $\Delta \Psi \mathrm{m}$. On the other hand, there was no notable change in the percentage of cells that lost their $\Delta \Psi \mathrm{m}$ between the LPS + ghrelin group and the LPS group, as well as between the ghrelin group and the control group, indicating that $\Delta \Psi \mathrm{m}$ in neutrophils was not affected by ghrelin whatsoever.

Detection of neutrophil by apoptosis TUNEL assay. Cell apoptosis was then examined using a TUNEL assay (Fig. 4). The TUNEL-positive rates were $74.00 \pm 2.65,23.00 \pm 3.46$, $29.33 \pm 3.284$ and $73.33 \pm 7.86 \%$ for the control, LPS, 
A
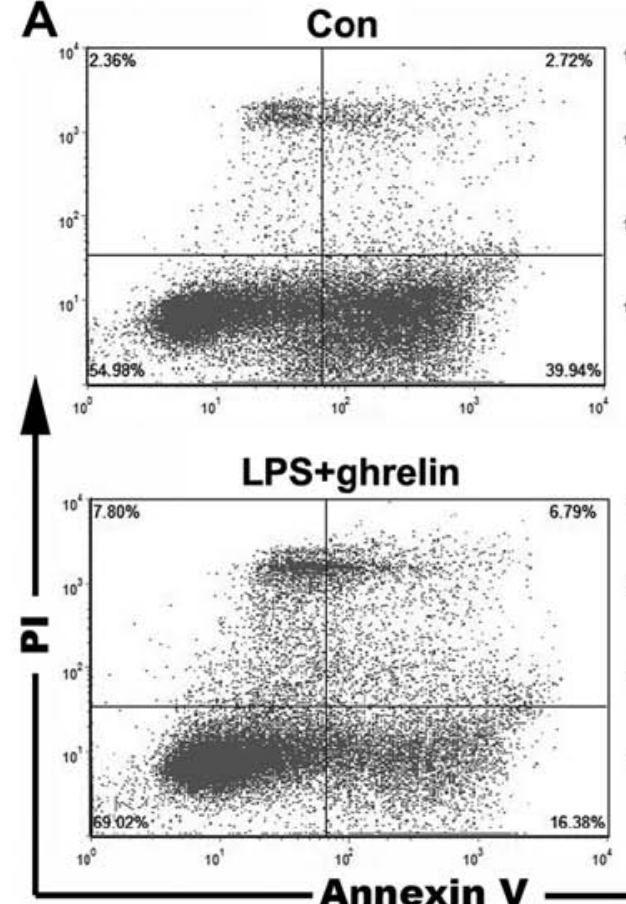

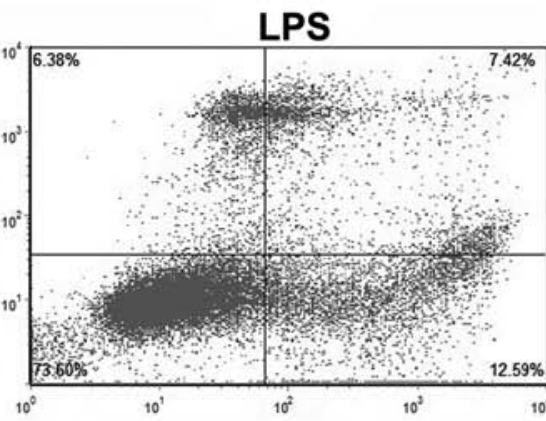

ghrelin

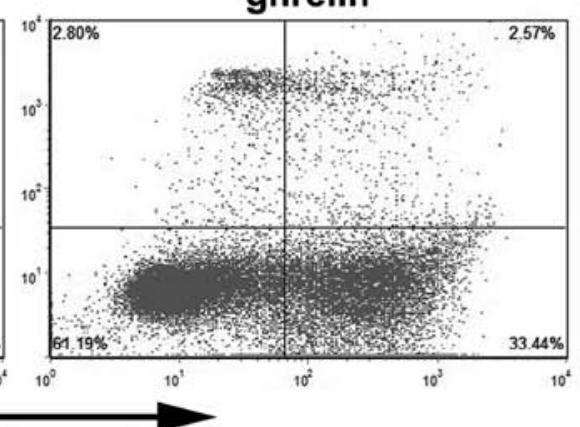

B

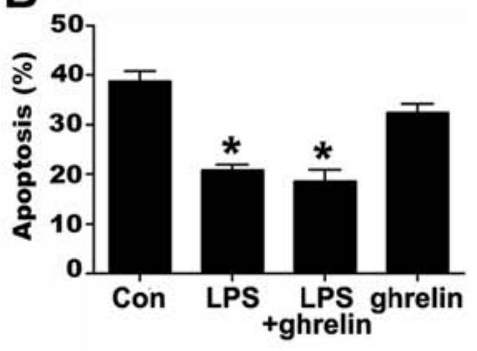

Figure 2. Cell apoptosis was measured by Annexin V-fluorescein isothiocyanate (FITC) staining. Human neutrophils were pre-treated with or without $100 \mathrm{nM}$ ghrelin for $1.5 \mathrm{~h}$ and then incubated with or without $100 \mathrm{ng} / \mathrm{ml}$ lipopolysaccharide (LPS) for $8 \mathrm{~h}$. (A) Dot graphs show the flowcytometric analysis of the apoptosis of the LPS- and ghrelin-treated neutrophils. (B) Quantitative analysis of neutrophils undergoing apoptosis. ${ }^{*} \mathrm{P}<0.05$ compared with the control.

A

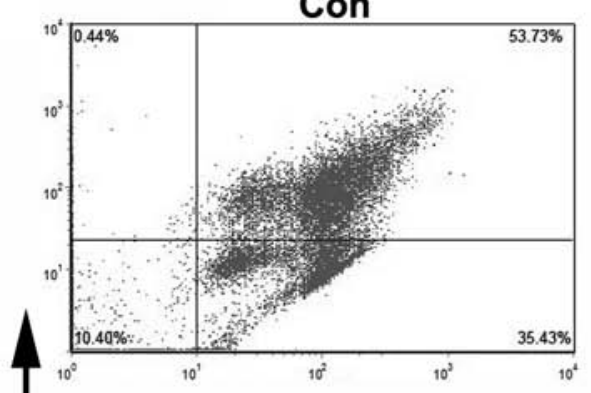

\section{LPS+ghrelin}

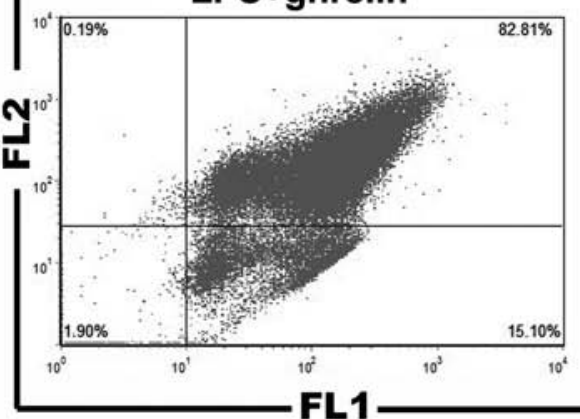

LPS

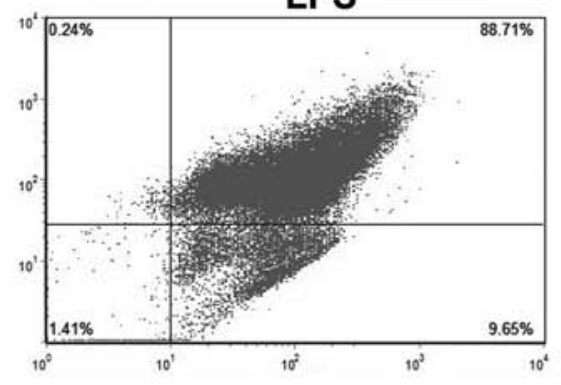

ghrelin

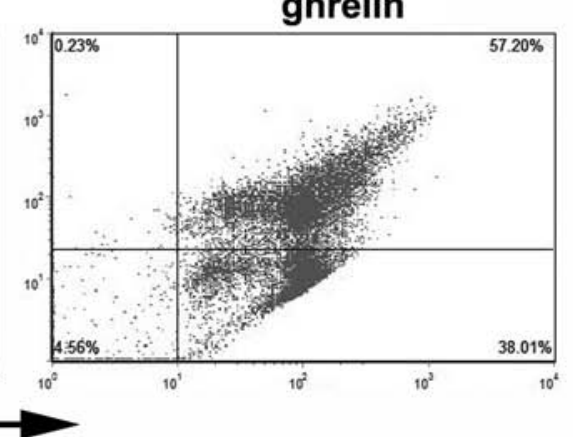

B

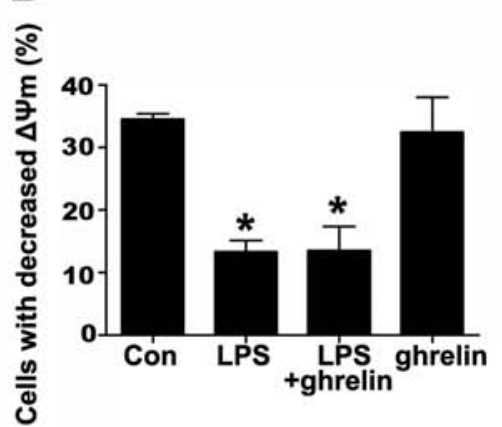

Figure 3. Effects of drugs on mitochondrial transmembrane potential $(\Delta \Psi \mathrm{m})$ in neutrophils. Cells were pre-treated with or without $100 \mathrm{nM}$ ghrelin for $1.5 \mathrm{~h}$ and then simulated with or without $100 \mathrm{ng} / \mathrm{ml}$ lipopolysaccharide (LPS) for $8 \mathrm{~h}$. (A) Dot graphs show the flowcytometric ananlysis of the mitochondrial transmembrane potential $(\Delta \Psi \mathrm{m})$ of the LPS- and ghrelin-treated neutrophils. $\Delta \Psi \mathrm{m}$ was then determined with the lipophilic dye, JC-1. (B) Quantitative analysis of neutrophils undergoing $\Delta \Psi \mathrm{m}$. ${ }^{*} \mathrm{P}<0.05$ compared with the control.

LPS + ghrelin and ghrelin groups, respectively. Our results revealed that LPS markedly decreased the number of TUNEL- positive cells compared with the controls. However, there were no apparent differences in the TUNEL positivity rates between 

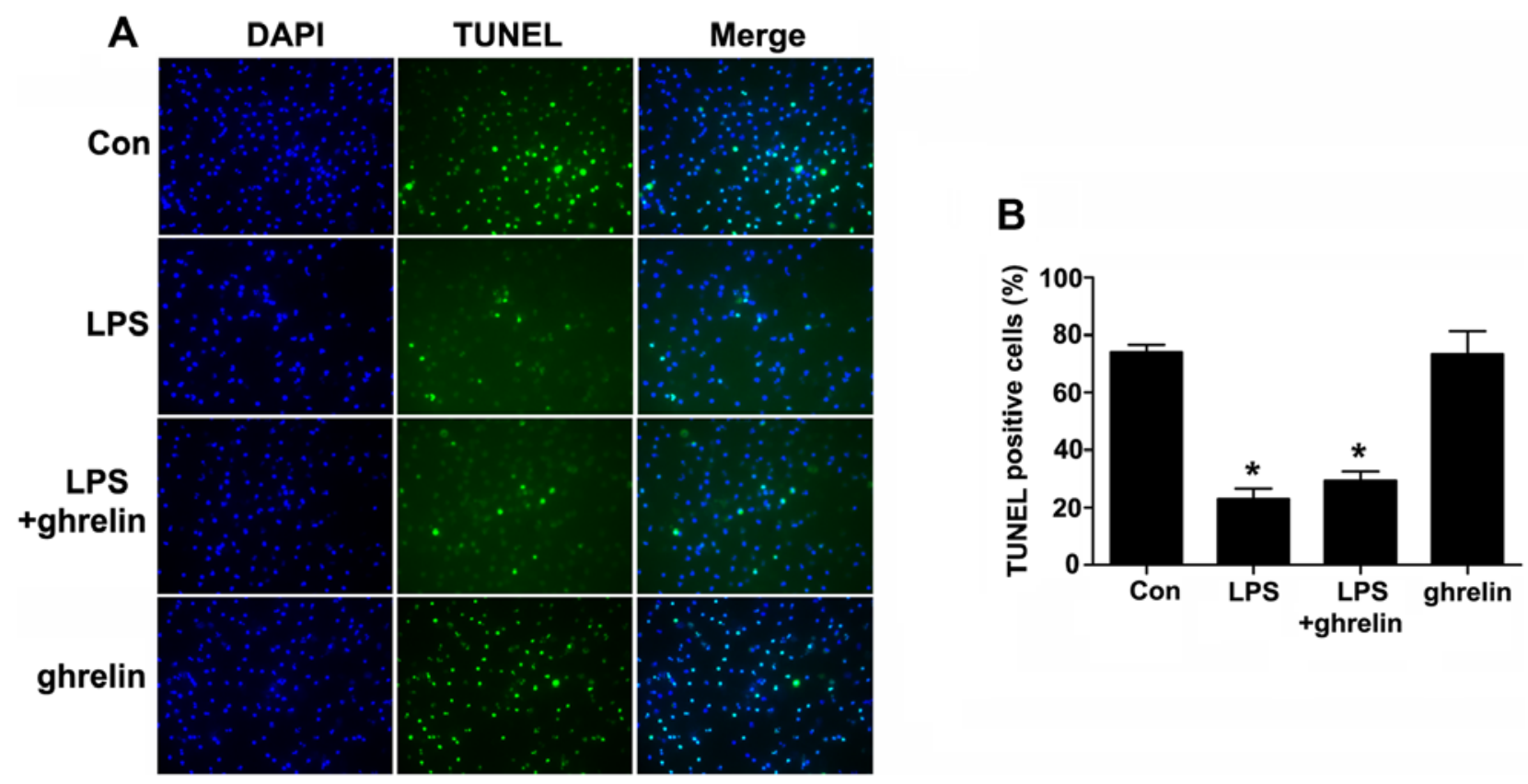

Figure 4. Terminal deoxynucleotidyl transferase (TdT)-mediated dUTP-biotin nick-end labeling (TUNEL) staining assay of neutrophil nucler degeneration. Neutrophils were incubated in serum-free medium for $8 \mathrm{~h}$, with pre-treatment with or without $100 \mathrm{nM}$ ghrelin for $1.5 \mathrm{~h}$, in the presence or absence of $100 \mathrm{ng} / \mathrm{ml}$ lipopolysaccharide (LPS). (A) Representative images demonstrate TUNEL-positive nuclei (green color) (x100 magnification). (B) Percentages (\%) of TUNELpositive cells relative to 4',6-diamidino-2-phenylindole (DAPI)-positive total nuclei are indicated in the histogram. ${ }^{*} \mathrm{P}<0.05$ compared with the control.

the LPS group and the LPS + ghrelin group, and between the control and ghrelin groups, indicating that treatment with ghrelin may not affect the incidence of neutrophil apoptosis.

Effects of ghrelin on the Bcl-2/Bax ratio and cleaved caspase-3 expression. To further investigate the molecular mechanisms responsible for the ability of ghrelin to induce neutrophil apoptosis, western blot analysis was carried out to determine the levels of key neutrophil intracellular $\mathrm{Bcl}-2$ family proteins and caspase activation following drug stimulation. It has been shown that Bcl-2 forms a heterodimeric complex with the apoptotic protein Bax, thereby neutralizing its apoptotic effects (26). Therefore, the ratio of $\mathrm{Bcl}-2 / \mathrm{Bax}$ is often considered as a decisive factor in determining whether cells will undergo death or survive. The values of the $\mathrm{Bcl}-2 / \mathrm{Bax}$ ratio were obtained by dividing the OD for Bcl-2 with the OD for Bax in a different group. We observed that stimulation of the neutrophils with LPS resulted in a noted dose-dependent rise in the Bcl-2/Bax ratio and in the downregulation of cleaved caspase-3 at the protein level (Fig. 5A and B), which favors anti-apoptosis. It should be noted that treatment with ghrelin at the concentration of $100 \mathrm{nM}$ appeared to hardly have any effect on the Bcl-2, Bax and cleaved caspase-3 expression levels after $8 \mathrm{~h}$, as the $\mathrm{Bcl}-2 / \mathrm{Bax}$ ratio and cleaved caspase-3 expression in the LPS + ghrelin group did not show significant changes when compared with the LPS group (Fig. 5C and D). To determine whether caspases (cysteine aspartic specific proteases) are involved in neutrophil apoptosis in the cells exposed to LPS or ghrelin, we also used immunofluorescence staining to evaluate active cleaved caspases- 3 levels in the cells. The data presented in Fig. 6 show a significant decrease in the levels of cleaved caspase-3 in the neutrophils stimulated with LPS. Notably, pre-treatment with ghrelin did not increase the cleavage of caspase- 3 as previously thought.

\section{Discussion}

The precise mechanisms repsonbilse for the development of ARDS are complex and are not yet clear; however, neutrophil apoptosis is one of the mechanisms involved $(1,10)$. Neutrophils, the most numerous immune cells in the first line of protection against invading microbes, are characterized by a very short lifespan (27); their normally short lifespan in the circulation can be manipulated and extended at sites of inflammation, to ensure that an appropriate host response is mounted in response to infection or tissue injury. However, uncontrolled neutrophil recruitment or inappropriate neutrophil longevity is pathophysiologically involved in the development of inflammatory diseases with unresolved neutrophilic inflammation (28). For instance, a delay in apoptosis may lead to the prolonged release of neutrophil products and direct tissue injury, thus participating in the development of ARDS (10). The pharmacological manipulation of such a defective resolution step is therefore an attractive avenue for the development of novel pro-resolution and anti-inflammatory treatments (29).

Ghrelin has gained attention for its broad range of roles in various biological systems (30). One of the most extensively studied characteristics is that ghrelin plays a positive role during the resolution phase of acute inflammation through the attenuation of the pro-inflammatory mediator response $(31,32)$, neutrophil infiltration $(18,22,32)$, and the enhancement of the phagocytosis of apoptotic cells (33), resulting in therapeutic benefits in pathological conditions associated with inflammation (16). Previous studies have suggested that ghrelin and 
A

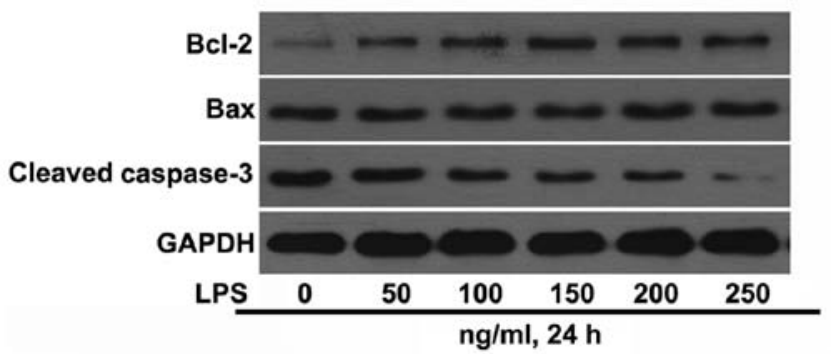

C

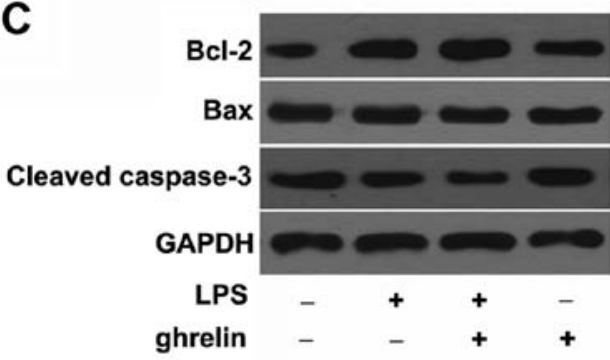

B

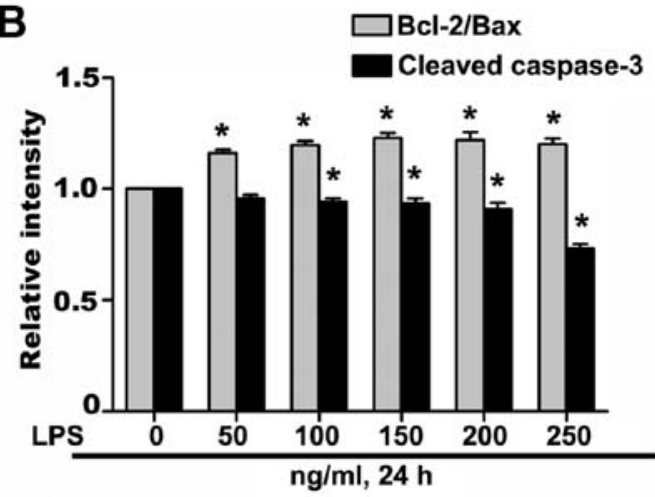

D

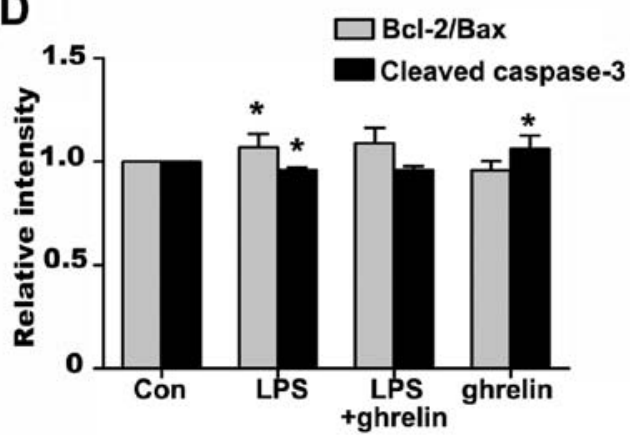

Figure 5. (A-D) Effects of lipopolysaccharide (LPS) or ghrelin on the expression of Bax, Bcl-2, cleaved caspase-3 and Bcl-2/Bax ratio in neutrophils by western blot analysis. The bar graphs represented densitometric results for Bcl-2/Bax and cleaved caspsase-3. " $\mathrm{P}<0.05$ compared with the control.

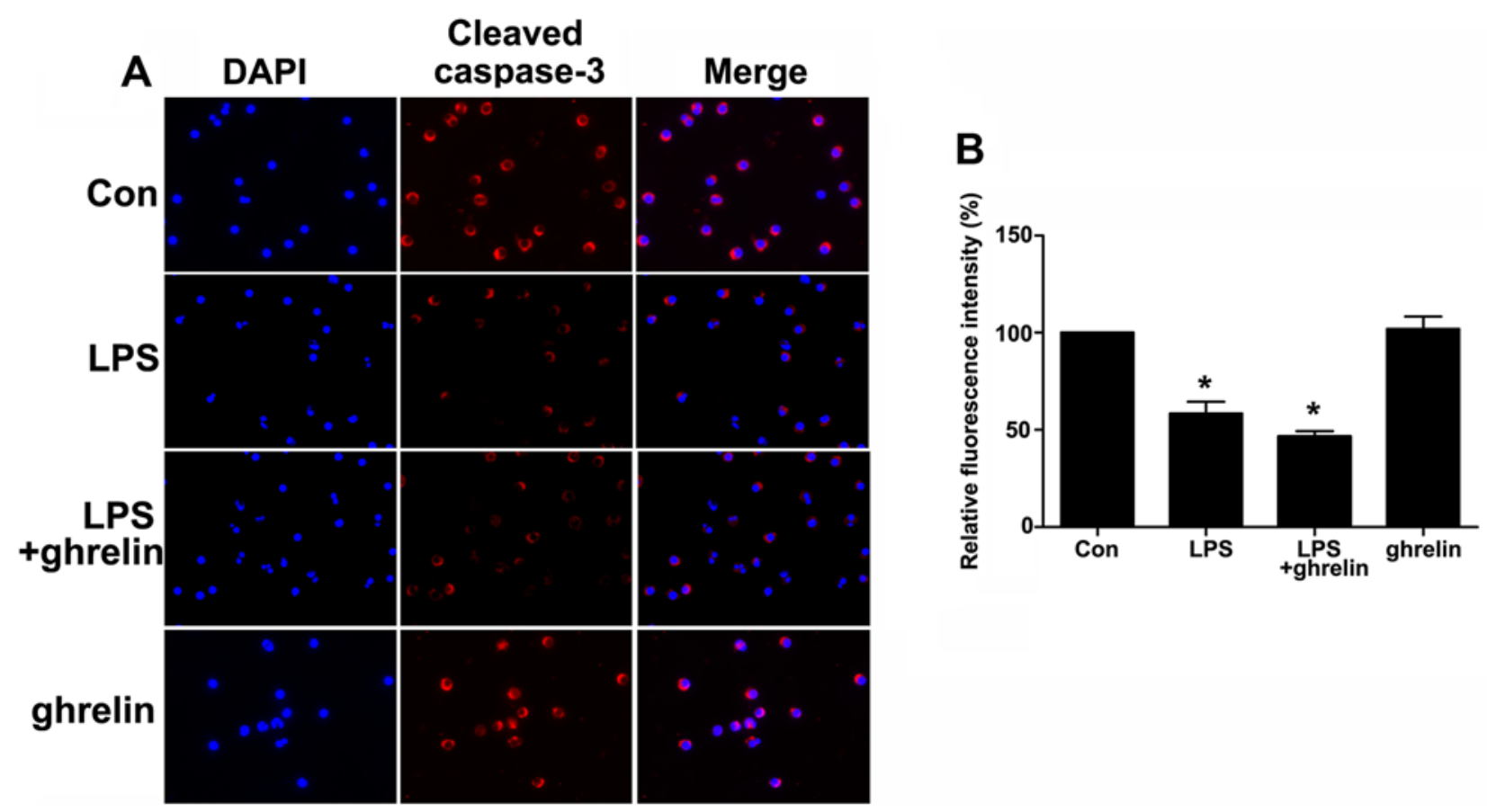

Figure 6. Immunofluorescence staining of cleaved caspase-3 expression in neutrophils following exposure to lipopolysaccharide (LPS) or ghrelin. (A) The red signal represents the cleaved caspase-3 levels (x200 magnification). (B) The graphs represent the means \pm SD of cleaved caspase- 3 fluorescence intensity. ${ }^{*} \mathrm{P}<0.05$ compared with the control.

ghrelin receptor are expressed in immune cells, including neutrophils $(34,35)$, alveolar macrophages and human lung tissue (36), indicating that ghrelin has direct modulatory effects on pulmonary function. Further studies have indicated that ghrelin displays anti-inflammatory properties in ARDS, leading to ameliorated pulmonary injury in rats with ARDS (20). In our previous study, we demonstrated that ghrelin inhibited apoptosis and enhanced the phagocytosis of alveolar macrophages, thus mitigating lung injury in septic ARDS (37). Therefore, we hypothesized that ghrelin has a potential modulatory role 
in neutrophil apoptosis for two reasons: first, ghrelin has been extensively reported to play a positive role in various inflammatory diseases; second, the ability of ghrelin to modulate apoptosis has been proven in several cell types previously $(22,38,39)$. In contrast to the induction of the apoptosis of endothelial and epithelial cells, the apoptosis of neutrophils is decreased during sepsis-induced ARDS (40). Thus, we originally predicted that ghrelin may act as a pro-apoptotic modulator of neutrophil apoptosis, subsequently relieving lung injury in the pathological process of ARDS. We believe that the careful analysis of neutrophil apoptosis in response to ghrelin treatment is warranted in order to better understand the mechanisms responsible for the resolution of inflammation after the administration of ghrelin. Our findings using flow cytometry demonstrated that stimulation of the cells with $100 \mathrm{ng} / \mathrm{ml}$ of LPS decreased the apoptotic ratio of the neutrophils, which was consistent with previous reports $(23,41)$. However, pre-treatment with $100 \mathrm{nM}$ ghrelin did not increase the apoptotic ratio (Fig. 2), indicating that ghrelin may not enhance the resolution of inflammation by promoting neutrophil apoptosis.

The apoptotic pathway is controlled by the balance of pro- vs. anti-apoptotic proteins from the Bcl-2 family, which together regulate mitochondrial outer membrane permeabilization and the release of pro-apoptotic substances from the mitochondria (42). Neutrophils also express Bcl-2 family members, the most relevant in regulating their survival $(42,43)$, which positively correlate with the neutrophil lifespan $(27,44)$. Our data clearly demonstrated that the ratio of Bcl-2/Bax markedly rose in the LPS-stimulated neutrophils, whereas we did not observe any notable changes in the ratio of Bcl-2/ Bax following pre-treatment with ghrelin (Fig. 5). The stabilization of $\Delta \Psi \mathrm{m}$ plays an essential role in the mitochondrial apoptotic pathway (45). Our study demonstrated that LPS can help sustain the stabilization of $\Delta \Psi \mathrm{m}$ to prolong the lifespan of neutrophils. However, treatment with ghrelin did not alter the $\Delta \Psi \mathrm{m}$, revealing for the first time that the anti-apoptotic effects of ghrelin through mitochondrial signaling do not occur in human neutrophils (Fig. 3). Using TUNEL assay, we also investigated nuclear morphological changes following drug treatment. Consistent with the results of flow cytometry and $\Delta \Psi \mathrm{m}$ assay, the results of TUNEL assay revealed that LPS markedly decreased the double-stranded cleavage of DNA, while treatment with ghrelin hardly had any influence on DNA double-stranded cleavage in neutrophils (Fig. 4).

Apoptosis can proceed via either an extrinsic pathway upon the ligation of a death receptor or an intrinsic pathway in response to cellular stress, chemotherapeutic agents, or ultraviolet damage (46). Both apoptotic pathways eventually act through the activation of enzymes known as caspases, which are responsible for the numerous morphological and biochemical changes in apoptosis (46). Cleaved caspase-3 has been proposed as a regulator of the neutrophil lifespan, as it is present in mature neutrophils and its expression correlates with increased apoptosis $(43,46)$. Consistent with the dependence on activated caspases for apoptosis, we observed that LPS caused a decrease in caspase-3 activation. We also observed that ghrelin had no influence on the cleavage of caspase-3, as pre-treatment with ghrelin did not alter the cleaved caspase- 3 expression level, as shown by western blot analysis or immunofluorescence staining (Figs. 5 and 6).
The important role of neutrophils in ARDS has been demonstrated in previous studies, in which the severity of lung injury was shown to decrease when neutrophils were eliminated $(10,40)$. Therefore, we originally predicted that ghrelin may be identified as a modulator of neutrophil apoptosis and sought insight into the underlying molecular mechanisms. Interestingly, we found that ghrelin neither abrogated the prosurvival influence of LPS that prolongs the neutrophil lifespan nor induced neutrophil apoptosis. It is not entirely clear why the effect of ghrelin on apoptosis varies between cell types; however, there is evidence to suggest that ghrelin indeed possesses anti- and pro-apoptotic functions towards different cells (47-50). For example, ghrelin has been previously shown to inhibit or induce the apoptosis of lung epithelial cells (22) and human colorectal adenocarcinoma cells (51). However, to the best of our knowledge, there is no previous available study characterizing the direct effects of ghrelin on the regulation of the neutrophil lifespan. In this study, we firstly reported that ghrelin neither inhibited nor induced neutrophil apoptosis, which was not consistent with our initial hypothesis.

We consider that there are several possible reasons to explain this result. To begin with, past studies have suggested that aside from the regulation of apoptosis, many other modulatory mechanisms also contribute to the pharmacological anti-inflammatory functions of ghrelin, varying from the inhibition of intracellular signaling cascades (52), to reduced cytokine production (52-54), an enhanced ability to eliminate invading microorganisms (37), and the inhibition of immunocyte apoptosis (55). Ghrelin may inhibit the production and release of cytokines, reactive oxygen species, antimicrobial and proteolytic granule proteins in neutrophils or other cells, and enhance the ability of neutrophils to engulf pathogens, rather than boosting neutrophil apoptosis. As is known to us, activated neutrophils synthesize chemokines and cytokines, which recruit and regulate the inflammatory response of other effector cells, including macrophages, $\mathrm{T}$ cells and neutrophils themselves $(3,56)$. For example, pro-inflammatory cytokines, such as interleukin (IL)-1 $\beta$, tumor necrosis factor (TNF)- $\alpha$, interferon (IFN)- $\mu$, and granulocyte/macrophage colony stimulating factor, can inhibit neutrophil apoptosis, whereas IL-10 counteracts this inhibition $(27,57)$. Taken together, the elimination of microorganisms by neutrophils can be regarded as a dynamic process, integrating the synthesis of granule proteins during differentiation, migration to sites of infection, phagocytosis and the killing of microorganisms, the modulation of other effector cells and, finally, apoptosis. We speculate that although ghrelin did not influence neutrophil apoptosis in vitro, it may indirectly affect neutrophil apoptosis by modulating the secretion of various chemokines and cytokines, phagocytosis, and other steps involved in the cascade of defense mechanisms in response to various physiological and pathological conditions in vivo, thus enhancing the resolution of inflammation and exerting therapeutic benefits. Second, this result may in part be explained by stimulated, inflammatory neutrophils having an altered sensitivity towards apoptosis-inducing stimuli. As an essential role to launch a first line of defense, substantial evidence has suggested that neutrophils experience an extensive change in gene expression and function, participating in previously unanticipated ways in a whole host of immune, inflammatory, and tissue remodeling responses that sustain life during stress (58). For example, TNF-related apoptosis-inducing ligand (TRAIL)-deficient mice 
show normal circulating neutrophil numbers and normal constitutive neutrophil apoptosis, but demonstrate impaired apoptosis when exposed to an inflammatory environment (59). Thus, we hypothesized that the in vitro freshly isolated and LPS-stimulated neutrophils may also alter their sensitivity toward ghrelin. Third, we adopted the concentration of $100 \mathrm{nM}$ ghrelin in this study as it was extensively accepted in previous literature $(60,61)$. As we mentioned above, the sensitivity toward apoptosis-inducing stimuli in neutrophils may vary from normal physiological conditions, and thus the apoptotic response to ghrelin may also be altered and the physiological concentration of $100 \mathrm{nM}$ ghrelin was possibly not intense enough to cause neutrophil apoptosis in the freshly isolated and LPS-stimulated neutrophils. Thus, dosedependent effects need to be investigated to better explain the observed anti-inflammatory effects and beneficial consequences of ghrelin administration in ARDS.

In conclusion, to the best of our knowledge, our findings demonstrate for the first time that ghrelin does not drive neutrophil apoptosis in a mitochondrial-dependent manner to override the LPS-induced delay of apoptosis. Thus, the identification of specific regulatory mechanisms responsible for the ability of ghrelin to ameliorate inflammation in ARDS warrants further investigation in future studies, helping to explain the beneficial effects of ghrelin administration in various pathological states associated with inflammation.

\section{Acknowledgements}

This study was supported by Grants from the Science and Technology Program of Guangzhou City of China (2014Y2-00136), the Science and Technology Program of Guangdong Province of China (2014A020212151) and the Science and Technology Program of Guangdong Province of China (2016A020216009).

\section{References}

1. Ranieri VM, Rubenfeld GD, Thompson BT, Ferguson ND, Caldwell E, Fan E, Camporota L and Slutsky AS; ARDS Definition Task Force: Acute respiratory distress syndrome: the berlin definition. JAMA 307: 2526-2533, 2012.

2. Nathan C: Neutrophils and immunity: challenges and opportunities. Nat Rev Immunol 6: 173-182, 2006.

3. Martin KR, Ohayon D and Witko-Sarsat V: Promoting apoptosis of neutrophils and phagocytosis by macrophages: novel strategies in the resolution of inflammation. Swiss Med Wkly 145: w14056, 2015

4. Ma HJ, Huang XL, Liu Y and Fan YM: Sulfur dioxide attenuates LPS-induced acute lung injury via enhancing polymorphonuclear neutrophil apoptosis. Acta Pharmacol Sin 33: 983-990, 2012.

5. Recher M, Malipiero U, Schaer DJ, Koedel U, Pfister HW, Birchler T, Petrausch U, Claus H, Gast H and Fontana A: Inhibition of meningitis-associated neutrophil apoptosis by TNF- $\alpha$ depends on functional PI3-kinase in monocytes. J Leukoc Biol 93: 259-266, 2013.

6. Walmsley SR, Print C, Farahi N, Peyssonnaux C, Johnson RS Cramer T, Sobolewski A, Condliffe AM, Cowburn AS, Johnson N and Chilvers ER: Hypoxia-induced neutrophil survival is mediated by HIF-1alpha-dependent NF-kappaB activity. J Exp Med 201: 105-115, 2005.

7. Pryde JG, Walker A, Rossi AG, Hannah S and Haslett C: Temperature-dependent arrest of neutrophil apoptosis. Failure of Bax insertion into mitochondria at 15 degrees $\mathrm{C}$ prevents the release of cytochrome c. J Biol Chem 275: 33574-33584, 2000.

8. Savill JS, Wyllie AH, Henson JE, Walport MJ, Henson PM and Haslett C: Macrophage phagocytosis of aging neutrophils in inflammation. Programmed cell death in the neutrophil leads to its recognition by macrophages. J Clin Invest 83: 865-875, 1989.

9. Simon HU: Targeting apoptosis in the control of inflammation. Eur Respir J Suppl 44 (Suppl 44): 20s-21s, 2003.
10. Fialkow L, Fochesatto Filho L, Bozzetti MC, Milani AR, Rodrigues Filho EM, Ladniuk RM, Pierozan P, de Moura RM, Prolla JC, Vachon E and Downey GP: Neutrophil apoptosis: a marker of disease severity in sepsis and sepsis-induced acute respiratory distress syndrome. Crit Care 10: R155, 2006.

11. Persson CG and Uller L: Increased lung neutrophil apoptosis and inflammation resolution. Eur Respir J 39: 789-790, author reply 790-791, 2012.

12. Hutcheson R, Terry R, Hutcheson B, Jadhav R, Chaplin J, Smith E, Barrington R, Proctor SD and Rocic P: miR-21-mediated decreased neutrophil apoptosis is a determinant of impaired coronary collateral growth in metabolic syndrome. Am J Physiol Heart Circ Physiol 308: H1323-H1335, 2015.

13. Elks PM, van Eeden FJ, Dixon G, Wang X, Reyes-Aldasoro CC, Ingham PW, Whyte MK, Walmsley SR and Renshaw SA: Activation of hypoxia-inducible factor- $1 \alpha$ (Hif- $1 \alpha$ ) delays inflammation resolution by reducing neutrophil apoptosis and reverse migration in a zebrafish inflammation model. Blood 118: 712-722, 2011.

14. Kolaczkowska E, Plytycz B, Arnold B, Piccard H and Opdenakker G: Increased cyclooxygenase activity impairs apoptosis of inflammatory neutrophils in mice lacking gelatinase $\mathrm{B} /$ matrix metalloproteinase-9. Immunology 128 (Suppl 1): e262-e274, 2009.

15. Koedel U, Frankenberg T, Kirschnek S, Obermaier B, Häcker H, Paul R and Häcker G: Apoptosis is essential for neutrophil functional shutdown and determines tissue damage in experimental pneumococcal meningitis. Plos Pathog 5: e1000461, 2009.

16. Baatar D, Patel K and Taub DD: The effects of ghrelin on inflammation and the immune system. Mol Cell Endocrinol 340: 44-58, 2011.

17. Dixit VD and Taub DD: Ghrelin and immunity: a young player in an old field. Exp Gerontol 40: 900-910, 2005.

18. Kodama T, Ashitani J, Matsumoto N, Kangawa K and Nakazato M: Ghrelin treatment suppresses neutrophil-dominant inflammation in airways of patients with chronic respiratory infection. Pulm Pharmacol Ther 21: 774-779, 2008.

19. Chang L, Zhao J, Yang J, Zhang Z, Du J and Tang C: Therapeutic effects of ghrelin on endotoxic shock in rats. Eur J Pharmacol 473: 171-176, 2003.

20. Wu R, Dong W, Zhou M, Zhang F, Marini CP, Ravikumar TS and Wang P: Ghrelin attenuates sepsis-induced acute lung injury and mortality in rats. Am J Respir Crit Care Med 176: 805-813, 2007.

21. Granado M, Priego T, Martín AI, Villanúa MA and López-Calderón A: Anti-inflammatory effect of the ghrelin agonist growth hormone-releasing peptide-2 (GHRP-2) in arthritic rats. Am J Physiol Endocrinol Metab 288: E486-E492, 2005.

22. Imazu Y, Yanagi S, Miyoshi K, Tsubouchi H, Yamashita S, Matsumoto N, Ashitani J, Kangawa K and Nakazato M: Ghrelin ameliorates bleomycin-induced acute lung injury by protecting alveolar epithelial cells and suppressing lung inflammation. Eur J Pharmacol 672: 153-158, 2011.

23. Jia SH, Parodo J, Kapus A, Rotstein OD and Marshall JC: Dynamic regulation of neutrophil survival through tyrosine phosphorylation or dephosphorylation of caspase-8. J Biol Chem 283: 5402-5413, 2008.

24. Pouliot M, Fiset ME, Massé M, Naccache PH and Borgeat P: Adenosine upregulates cyclooxygenase-2 in human granulocytes: impact on the balance of eicosanoid generation. J Immunol 169: 5279-5286, 2002.

25. Hirsch T, Marzo I and Kroemer G: Role of the mitochondrial permeability transition pore in apoptosis. Biosci Rep 17: 67-76, 1997.

26. Wang K, Gross A, Waksman G and Korsmeyer SJ: Mutagenesis of the $\mathrm{BH} 3$ domain of BAX identifies residues critical for dimerization and killing. Mol Cell Biol 18: 6083-6089, 1998.

27. Akgul C, Moulding DA and Edwards SW: Molecular control of neutrophil apoptosis. FEBS Lett 487: 318-322, 2001

28. Nishiura H, Zhao R, Chen J, Taniguchi K and Yamamoto T: Involvement of regional neutrophil apoptosis promotion by ribosomal protein S19 oligomers in resolution of experimental acute inflammation. Pathol Int 63: 581-590, 2013

29. Jančinová V, Perečko T, Nosál R, Mihalová D, Bauerová K and Drábiková K: Pharmacological regulation of neutrophil activity and apoptosis: contribution to new strategy for modulation of inflammatory processes. Interdiscip Toxicol 4: 11-14, 2011.

30. Khatib MN, Shankar A, Kirubakaran R, Agho K, Simkhada P, Gaidhane S, Saxena D, B U, Gode D, Gaidhane A and Zahiruddin SQ: Effect of ghrelin on mortality and cardiovascular outcomes in experimental rat and mice models of heart failure: a systematic review and meta-analysis. PLoS One 10: e0126697, 2015. 
31. Wang D, Wang H, Luo P, Hwang A, Sun D, Wang Y,Zhang Z, Liu N Wang S, Li C and Cao F: Effects of ghrelin on homocysteine-induced dysfunction and inflammatory response in rat cardiac microvascular endothelial cells. Cell Biol Int 36: 511-517, 2012.

32. Sehirli O, Sener E, Sener G, Cetinel S, Erzik C and Yeğen BC: Ghrelin improves burn-induced multiple organ injury by depressing neutrophil infiltration and the release of pro-inflammatory cytokines. Peptides 29: 1231-1240, 2008.

33. Yada T, Kaiya H, Mutoh K, Azuma T, Hyodo S and Kangawa K: Ghrelin stimulates phagocytosis and superoxide production in fish leukocytes. J Endocrinol 189: 57-65, 2006.

34. Hattori N, Saito T, Yagyu T, Jiang BH, Kitagawa $\mathrm{K}$ and Inagaki $\mathrm{C}$ : $\mathrm{GH}, \mathrm{GH}$ receptor, $\mathrm{GH}$ secretagogue receptor, and ghrelin expression in human T cells, B cells, and neutrophils. J Clin Endocrinol Metab 86: 4284-4291, 2001.

35. Hattori N: Expression, regulation and biological actions of growth hormone $(\mathrm{GH})$ and ghrelin in the immune system. Growth Horm IGF Res 19: 187-197, 2009.

36. Volante M, Fulcheri E, Allìa E, Cerrato M, Pucci A and Papotti M: Ghrelin expression in fetal, infant, and adult human lung. J Histochem Cytochem 50: 1013-21, 2012.

37. Li B, Zeng M, He W, Huang $X$, Luo L, Zhang $H$ and Deng DY: Ghrelin protects alveolar macrophages against lipopolysaccharide-induced apoptosis through growth hormone secretagogue receptor 1a-dependent c-Jun $\mathrm{N}$-terminal kinase and $\mathrm{Wnt} / \beta$-catenin signaling and suppresses lung inflammation. Endocrinology 156: 203-217, 2015.

38. Lee JY, Chung H, Yoo YS, Oh YJ, Oh TH, Park S and Yune TY: Inhibition of apoptotic cell death by ghrelin improves functional recovery after spinal cord injury. Endocrinology 151: 3815-3826 2010.

39. Baldanzi G, Filigheddu N, Cutrupi S, Catapano F, Bonissoni S, Fubini A, Malan D, Baj G, Granata R, Broglio F, et al: Ghrelin and des-acyl ghrelin inhibit cell death in cardiomyocytes and endothelial cells through ERK1/2 and PI 3-kinase/AKT. J Cell Biol 159: 1029-1037, 2002.

40. Wang H, Xu L, Zhao J, Wang D, Guo R, Wang J, Gong W, Liu T, Zhang Y and Dong L: Regulatory mechanism of pyrrolidine dithiocarbamate is mediated by nuclear factor- $\kappa \mathrm{B}$ and inhibits neutrophil accumulation in ARDS mice. Exp Ther Med 8 614-622, 2014

41. Turina M, Miller FN, Tucker C and Polk HC: Effects of hyperglycemia, hyperinsulinemia, and hyperosmolarity on neutrophil apoptosis. Surg Infect (Larchmt) 7: 111-121, 2006.

42. Croker BA, O'Donnell JA, Nowell CJ, Metcalf D, Dewson G, Campbell KJ, Rogers KL, Hu Y, Smyth GK, Zhang JG, et al: Fas-mediated neutrophil apoptosis is accelerated by Bid, Bak and Bax and inhibited by Bcl-2 and Mcl-1. Proc Natl Acad Sci USA 108: 13135-13140, 2011.

43. Maianski NA, Roos D and Kuijpers TW: Bid truncation, bid/bax targeting to the mitochondria, and caspase activation associated with neutrophil apoptosis are inhibited by granulocyte colony-stimulating factor. J Immunol 172: 7024-7030, 2004

44. Edwards SW, Moulding DA, Derouet $M$ and Moots RJ Regulation of neutrophil apoptosis. Chem Immunol Allergy 83: 204-224, 2003

45. Maianski NA, Geissler J, Srinivasula SM, Alnemri ES, Roos D and Kuijpers TW: Functional characterization of mitochondria in neutrophils: a role restricted to apoptosis. Cell Death Differ 11: $143-153,2004$.

46. Espino J, Bejarano I, Redondo PC, Rosado JA, Barriga C, Reiter RJ, Pariente JA and Rodríguez AB: Melatonin reduces apoptosis induced by calcium signaling in human leukocytes: evidence for the involvement of mitochondria and Bax activation. J Membr Biol 233: 105-118, 2010.
47. Cassoni P, Papotti M, Ghè C, Catapano F, Sapino A, Graziani A, Deghenghi R, Reissmann T, Ghigo E and Muccioli G: Identification, characterization, and biological activity of specific receptors for natural (ghrelin) and synthetic growth hormone secretagogues and analogs in human breast carcinomas and cell lines. J Clin Endocrinol Metab 86: 1738-1745, 2001.

48. Ghè C, Cassoni P, Catapano F, Marrocco T, Deghenghi R, Ghigo E, Muccioli G and Papotti M: The antiproliferative effect of synthetic peptidyl GH secretagogues in human CALU-1 lung carcinoma cells. Endocrinology 143: 484-491, 2002.

49. Cassoni P, Ghé C, Marrocco T, Tarabra E, Allia E, Catapano F, Deghenghi R, Ghigo E, Papotti M and Muccioli G: Expression of ghrelin and biological activity of specific receptors for ghrelin and des-acyl ghrelin in human prostate neoplasms and related cell lines. Eur J Endocrinol 150: 173-84, 2004.

50. Zhang GG, Cai HQ, Li YH, Sui YB, Zhang JS, Chang JR, Ning M, Wu Y, Tang CS, Qi YF and Yin XH: Ghrelin protects heart against ERS-induced injury and apoptosis by activating AMP-activated protein kinase. Peptides 48: 156-165, 2013.

51. Bonfili L, Cuccioloni M, Cecarini V, Mozzicafreddo M, Palermo FA, Cocci P, Angeletti M and Eleuteri AM: Ghrelin induces apoptosis in colon adenocarcinoma cells via proteasome inhibition and autophagy induction. Apoptosis 20: 1188-200, 2013.

52. Li WG, Gavrila D, Liu X, Wang L, Gunnlaugsson S, Stoll LL, McCormick ML, Sigmund CD, Tang $C$ and Weintraub NL: Ghrelin inhibits proinflammatory responses and nuclear factor-kappaB activation in human endothelial cells. Circulation 109: 2221-2226, 2004.

53. Dixit VD, Schaffer EM, Pyle RS, Collins GD, Sakthivel SK, Palaniappan R, Lillard JW Jr and Taub DD: Ghrelin inhibits leptin- and activation-induced proinflammatory cytokine expression by human monocytes and T cells. J Clin Invest 114: 57-66, 2004.

54. Xia Q, Pang W, Pan H, Zheng Y, Kang JS and Zhu SG: Effects of ghrelin on the proliferation and secretion of splenic $\mathrm{T}$ lymphocytes in mice. Regul Pept 122: 173-178, 2004.

55. Zhu J, Zheng C, Chen J, Luo J, Su B, Huang Y, Su W, Li Z and Cui T: Ghrelin protects human umbilical vein endothelial cells against high glucose-induced apoptosis via mTOR/P70S6K signaling pathway. Peptides 52: 23-28, 2014.

56. Scapini P and Cassatella MA: Social networking of human neutrophils within the immune system. Blood 124: 710-719, 2014.

57. Jia SH, Li Y, Parodo J, Kapus A, Fan L, Rotstein OD and Marshall JC: Pre-B cell colony-enhancing factor inhibits neutrophil apoptosis in experimental inflammation and clinical sepsis. J Clin Invest 113: 1318-1327, 2004.

58. Burton JL, Madsen SA, Chang LC, Weber PS, Buckham KR, van Dorp R, Hickey MC and Earley B: Gene expression signatures in neutrophils exposed to glucocorticoids: a new paradigm to help explain 'neutrophil dysfunction' in parturient dairy cows. Vet Immunol Immunopathol 105: 197-219, 2005.

59. McGrath EE, Marriott HM, Lawrie A, Francis SE, Sabroe I, Renshaw SA, Dockrell DH and Whyte MK: TNF-related apoptosis-inducing ligand (TRAIL) regulates inflammatory neutrophil apoptosis and enhances resolution of inflammation. $\mathbf{J}$ Leukoc Biol 90: 855-865, 2011.

60. Chung H, Kim E, Lee DH, Seo S, Ju S, Lee D, Kim H and Park S: Ghrelin inhibits apoptosis in hypothalamic neuronal cells during oxygen-glucose deprivation. Endocrinology 148: 148-159, 2007.

61. Kim SW, Her SJ, Park SJ, Kim D, Park KS, Lee HK, Han BH, Kim MS, Shin CS and Kim SY: Ghrelin stimulates proliferation and differentiation and inhibits apoptosis in osteoblastic MC3T3-E1 cells. Bone 37: 359-369, 2005. 